\title{
Hypomethylating agents for treatment and prevention of relapse after allogeneic blood stem cell transplantation
}

\author{
Thomas Schroeder $^{1} \cdot$ Christina Rautenberg $^{1} \cdot$ Rainer Haas $^{1}$ - Ulrich Germing ${ }^{1} \cdot$ Guido Kobbe $^{1}$ \\ Received: 8 October 2017 / Accepted: 8 November 2017 / Published online: 15 November 2017 \\ (c) The Japanese Society of Hematology 2017
}

\begin{abstract}
Despite the curative potential of allogeneic stem cell transplantation (allo-SCT) in patients with acute myeloid leukemia (AML) and myelodysplastic syndromes (MDS), many patients will relapse. Until recently therapeutic options mainly consisted of palliative care, chemotherapy, donor lymphocyte infusions and second transplantation in selected cases. Still many patients either do not tolerate intensive therapies or do not achieve durable remissions and will finally succumb. Given this unmet medical need the hypomethylating agents (HMA), Azacitidine (Aza) and Decitabine (DAC) have been tested as salvage therapy in patients with myeloid malignancies relapsing after allo-SCT. Furthermore, they have also been incorporated into prophylactic and pre-emptive approaches to avoid haematological relapse. In this review, we summarize the evidence from retrospective studies but also from a few prospective trials regarding the use of HMA after transplant. To aid clinicians in their daily clinical practice, we also comment on some practical aspects such as dosing and schedule, the choice of HMA and the use of complementary cellular therapies. Finally, this review also gives an overview on potential mechanisms mediating the efficacy of HMA after transplant as well as ongoing preclinical research and clinical activities aiming to further improve this treatment approach.
\end{abstract}

Keywords Myelodysplastic syndromes $\cdot$ Acute myeloid leukemia $\cdot$ Allogeneic transplantation $\cdot$ Relapse $\cdot$ Maintenance . Decitabine · Azacitidine

\section{Introduction}

Allogeneic blood stem cell transplantation (allo-SCT) is a potentially curative treatment option for many patients with acute myeloid leukemia (AML) and represents the only chance for long-term survival in patients with myelodysplastic syndromes (MDS) [1]. In the past, several improvements including donor selection, immunosuppression and supportive care have been made to reduce non-relapse mortality. In addition, the introduction of reduced toxicity conditioning has broadened the access for more, in particular older patients to this treatment option [2].

Thomas Schroeder

thomas.schroeder@med.uni-duesseldorf.de

1 Department of Hematology, Oncology and Clinical Immunology, Medical Faculty, University of Duesseldorf, Moorenstr. 5, 40225 Düsseldorf, Germany
In contrast to this, relapse still represents the main cause of treatment failure and is associated with a poor prognosis. The principles of treatment in this challenging situation are to reduce the disease burden on the one hand and to induce an allogeneic immune reaction on the other hand to achieve long-term disease control. Traditionally, treatment options were limited and have generally consisted of palliative care, low-dose or intensive chemotherapy as well as cellular therapies such as donor lymphocyte infusions [3] and second transplantation in selected cases. Still, the fact that many patients can either not tolerate intensive therapies or are refractory to these conventional interventions indicates the relevant need for novel treatment approaches [4]. Ideally, such a therapy mediates direct antileukemic effects and strengthens the graft-versus-leukemia $(\mathrm{GvL})$ reaction, while on the other hand is not associated with an extensive risk for severe graft-versus-host disease (GvHD) and offers an acceptable toxicity profile. 
Given their balance between efficacy and moderate toxicity, the two hypomethylating agents (HMA) Azacitidine (Aza) and Decitabine (DAC) might meet many of these demands. Both are licensed and usually employed for the treatment of older patients with AML and MDS not eligible for intensive therapies [5-8]. Taking this into account, these two substances have also been tested in the post-transplant period.

In this review, we aim to summarize the current literature reporting on the use of Aza and DAC to prevent or to treat relapse of myeloid malignancies after allo-SCT. Besides an overview about ongoing research and clinical studies in this field, we also address practical issues regarding the use of these two HMA after transplant.

\section{Treatment of relapse with HMA}

\section{Azacitidine for the treatment of relapse}

In the absence of realistic treatment alternatives, we treated the first patient with early relapse of an AML evolved from MDS after allo-SCT with Aza and DLI in 2007 [9]. Following this combined pharmacological and cell-based approach, this woman achieved a complete remission (CR) and our observation presented the starting point for several retrospective studies reporting on the use of Aza as salvage therapy for relapse of myeloid malignancies after allo-SCT in a limited number of patients [10-12]. These data built the rationale for the first prospective multicenter trial (AZARELA, Eudra-CT 2007-004860-37) [13], where Aza was administered as first intervention for relapse and DLI were scheduled after every second Aza cycle.

All 30 patients included in this trial had hematologic relapse of AML $(n=28,92 \%)$ or MDS and MDS-MPS $(n=2,8 \%)$ in median 175 days after transplantation. They received a median of 3 courses of Aza (range 1-8) and 22 patients (73\%) finally received at least one DLI. This treatment resulted in an overall response rate of $30 \%$ including 7 patients (23\%) achieving CR and 2 patients (7\%) partial remission (PR). These remissions were durable in 5 of 7 patients lasting for a median of 777 days (range 461-890). One of these patients remains in ongoing remission without any further antileukemic treatment for 56 months until now. The finding that this therapy was in particular effective in patients with high-risk cytogenetics such as complex karyotype is in accordance with its primary indication in the non-transplant setting and gave already an early hint, which patients might benefit from this approach. This efficacy was not counterbalanced by an excess of toxicity and compared well if not better with other treatment options. Indeed, the rate and severity of GvHD as well as toxicities following the treatment with Aza and DLI were rather low and mild.
Altogether, this prospective study confirmed the observation from the retrospective reports that the combination of Aza and DLI could by a safe and effective treatment alternative for patients with myeloid malignancies who relapse after allo-SCT.

Table 1 summarizes the publications regarding the use of Aza as salvage treatment for relapse after allo-SCT: until now a total of 601 patients with AML, MDS and other related myeloid malignancies have been published with varying schedules and dosages of Aza. These included 3 prospective, non-randomized trials and the majority of patients reported retrospectively [9-25]. Furthermore, Aza was the first treatment of relapse and combined with DLI in some of these patients, while other patients had previously received other salvage therapies or did not receive DLI. This heterogeneity of treatment strategies explains CR rates and overall survival (OS, not given in details in a relevant number of many studies) ranging from 14 to $75 \%$ and from 12 to $80 \%$ after treatment with Aza.

Furthermore, as a consequence of this heterogeneity and limited number of patients in most of these analyses, the reproducible identification of factors predictive for response and long-term survival was not possible. For this purpose, two larger retrospective surveys were performed. In the first one, we analysed the outcome of 154 patients with hematologic $(88 \%)$ or molecular $(12 \%)$ relapse of AML or MDS after allo-HSCT. All were treated with Aza (median 4 courses; range 4-14) and DLI (administered to 105 patients, $68 \%$ ) either as first (93\%) or later (7\%) salvage approach at 12 transplant centres participating in the german cooperative transplant study group [22]. The size of this patient group and the quality of data provided by the participating centres enabled us to identify patients who may benefit most from the combination of Aza and DLI. Multivariate analyses carved out that the diagnosis of MDS and detection of relapse at a molecular stage were significantly predictive for the likelihood to achieve CR. In congruency with this, a low disease burden (molecular relapse or bone marrow blast count $<13 \%$ ) at the time of relapse and the diagnosis of MDS were also significant predictors for a longer overall survival [22].

These issues were also addressed in another retrospective analysis of a similar-sized patient group $(n=181)$ by Craddock et al. within the EBMT [16]. They also identified the diagnosis of MDS instead of AML and in addition transplantation in remission as predictors for response. Confirming our results with regard to overall survival disease burden defined by the bone marrow blast count (cut-off 20\%) at the time of relapse turned out to be predictive in multivariate analysis. Furthermore, a longer interval between allo-SCT and relapse (cut-offs 6 and 12 months) was also associated with a better outcome. These variables were included into a so-called AZA Relapse Prognostic Score (ARPS), which 


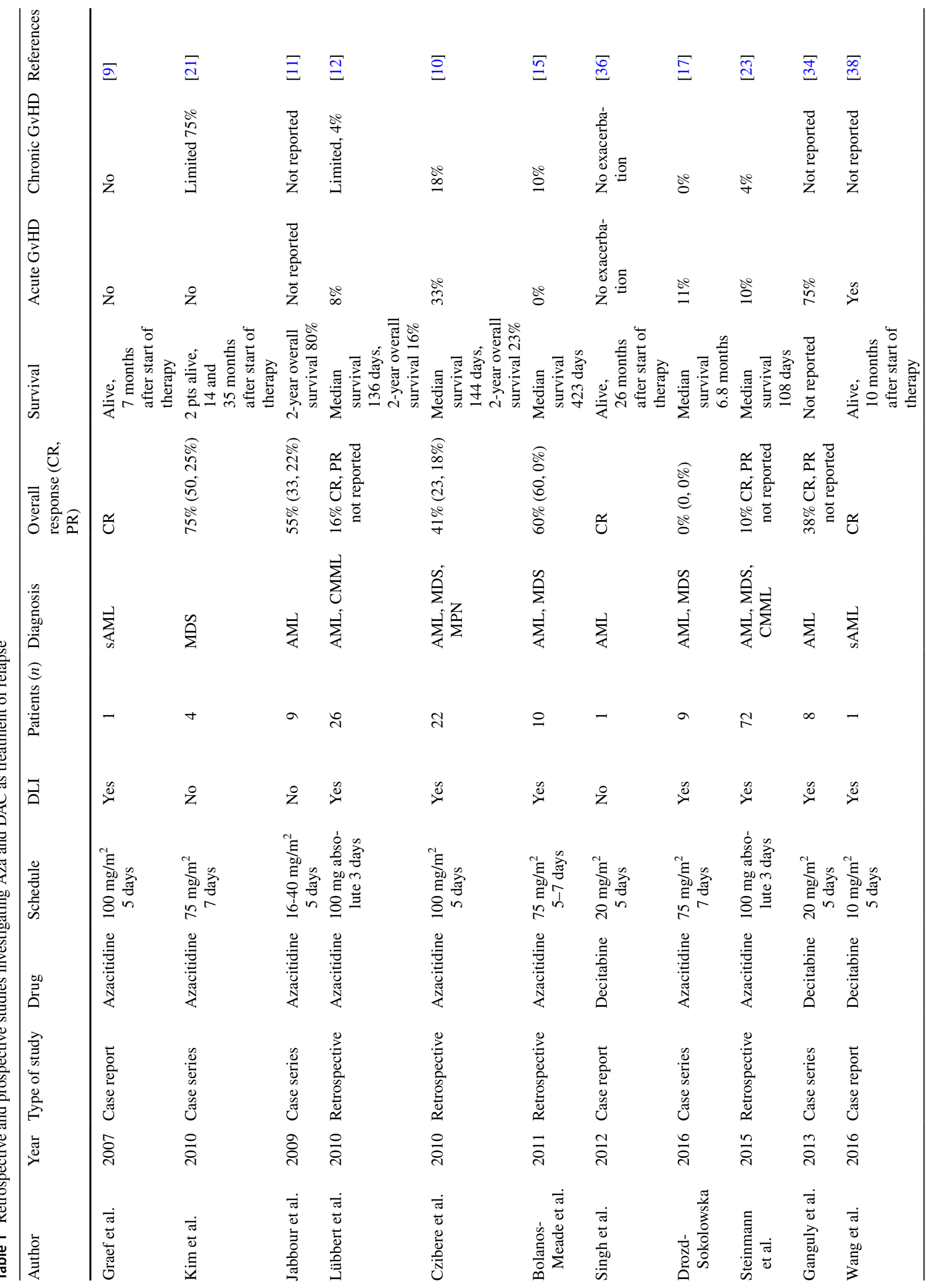




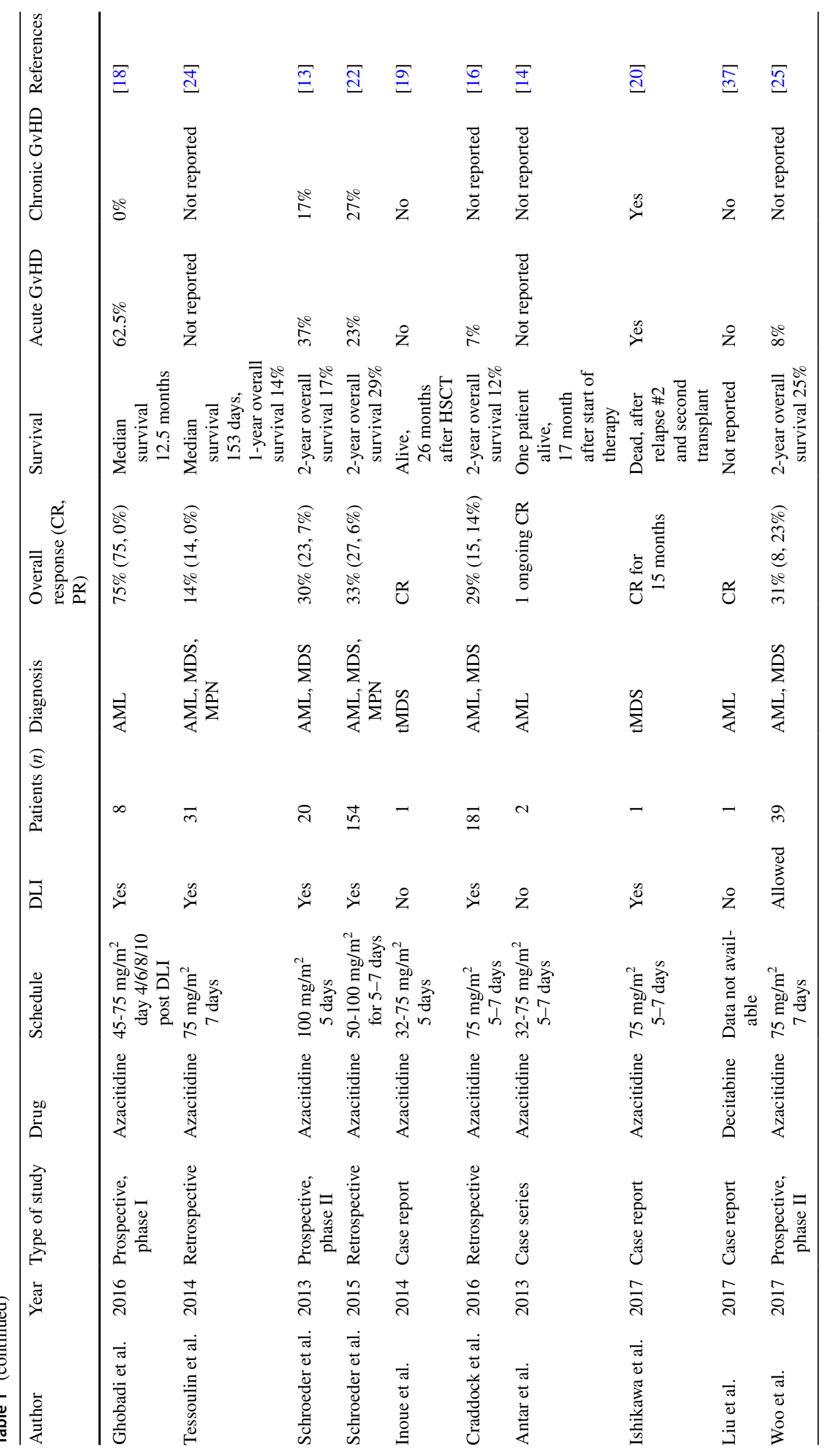


could clearly divide their cohort in 3 prognostically different subgroups. Still, this score has not been validated in an independent cohort yet and has only been tested in patients with haematological, but not molecular relapse due to the inclusion criteria of this registry-based analysis. In the light of the continuously optimized molecular methods to monitor minimal residual disease (MRD) and to guide MRD-triggered interventions after transplant, a cut-off of $20 \%$ BM blasts cast the practicability of this score for real life into doubt.

During the last years, several molecular alterations, mostly mutations have been unraveled in patients with MDS and AML [26, 27]. These mutations have substantially improved our pathophysiological understanding and can augment the outcome prediction after conventional therapy as well as after allo-SCT. As many of the identified mutations affect the so-called "epigenetic machinery", it was assumed that such mutations could potentially serve as predictors for response and survival after HMA treatment. Unfortunately, this has not proven to be the case in elderly patients treated with HMA, but not undergoing alloSCT [28-30]. In the context of HMA as salvage therapy after allo-SCT, one group has investigated this aspect so far. Woo and colleagues recently reported their results in 21 Aza-treated patients using a targeted 54 NGS gene panel. In their analysis, TP53 mutations were significantly associated with poor responsiveness to Aza and inferior survival, while the opposite applies by trend to TET2 mutations [31]. Nevertheless, the number of patients is too small to draw any conclusions asking for validation in larger patient groups.

Despite these controversies and the ongoing research including the search for biomarkers, the two large analyses together with the prospective and retrospective studies have univocally shown that the combination of Aza and DLI is of therapeutic value for patients relapsing after allo-SCT. Therefore, this approach has been incorporated as a treatment option for these patients in the current recommendations of the EBMT and the European Leukemia Net [32,33].

\section{Decitabine for the treatment of relapse}

DAC is the second HMA, which is approved in Europe for the treatment of elderly patients with AML. Furthermore, in the USA, it is also available for the treatment of patients with MDS. In the post-transplant setting, the literature reporting on the use of DAC as salvage therapy for relapse after alloSCT was restricted to a total of 11 patients reported so far (Table 1). With 6 of the treated patients achieving a complete remission, these case series suggested that DAC might also have some efficacy in patients with myeloid malignancies relapsing after allo-SCT [34-38]. Results from prospective trials investigating DAC as salvage therapy for relapse after allo-SCT have not been published so far and, to the best of our knowledge, will also not be available in the near future. Again, this prompted us to perform a retrospective survey on the use of DAC as salvage within the German Cooperative Transplant Study group. Hereby, we were able to analyse data of 36 patients with haematological $(n=35)$ or molecular relapse $(n=1)$, who received DAC as first salvage therapy (44\%) or after 1-5 previous lines of salvage therapy. As a result, CR rate was $23 \%(n=6,17 \%)$ including 3 patients within the first-line group. Of particular interest, 3 patients receiving DAC as second-line treatment after Aza (2 patients with failure, 1 patient with intolerability) also achieved CR. The 2-year OS rate was $11 \pm 6 \%$ without any difference between first-line and pretreated patients [39].

Together with an acceptable toxicity profile, these data suggest that also the second HMA DAC exerts clinical efficacy and can induce durable remissions in individual patients. Prospective trials are warranted to confirm that DAC may be an alternative to Aza or even a second choice after Aza failure.

\section{HMA for the prevention of relapse}

\section{Prophylactic versus pre-emptive therapy with HMA after allo-SCT}

As mentioned above, HMA can induce long-term remissions in a relevant number of patients with relapse after alloSCT. Nevertheless, the results of our analysis and the results from Craddock et al. showed that the success of HMA after transplant directly correlates with disease burden. Thus, it is rather better to avoid than to treat relapse or if not possible to start therapy at the lowest level of measurable disease. Approaches to reduce the risk of frank AML or MDS relapse following allo-SCT can be separated into prophylactic and pre-emptive strategies. Prophylactic approaches can be further subdivided into maintenance or consolidation therapies. While the former means a continuous therapy until disease progression or intolerability, the latter represents a therapy phase defined by a limited time interval and/or number of treatment cycles (Fig. 1).

Prophylactic treatment strategies aim to directly eliminate residual malignant cells, which cannot be detected with the currently available monitoring techniques. In addition, even if prophylactic treatment on its own fails to eradicate these undetectable, but present malignant cells, it may help to control disease activity until the donor immune system is sufficiently reconstituted to mediate the desired GvL effect. For this reason, prophylaxis concepts after allo-SCT not only incorporating HMA but also other compounds such as tyrosine kinase inhibitors are currently tested. However, in this context some concerns should be taken into account. Generally, some patients with high-risk myeloid malignancies will not require post-transplant cytotoxic therapy to achieve 


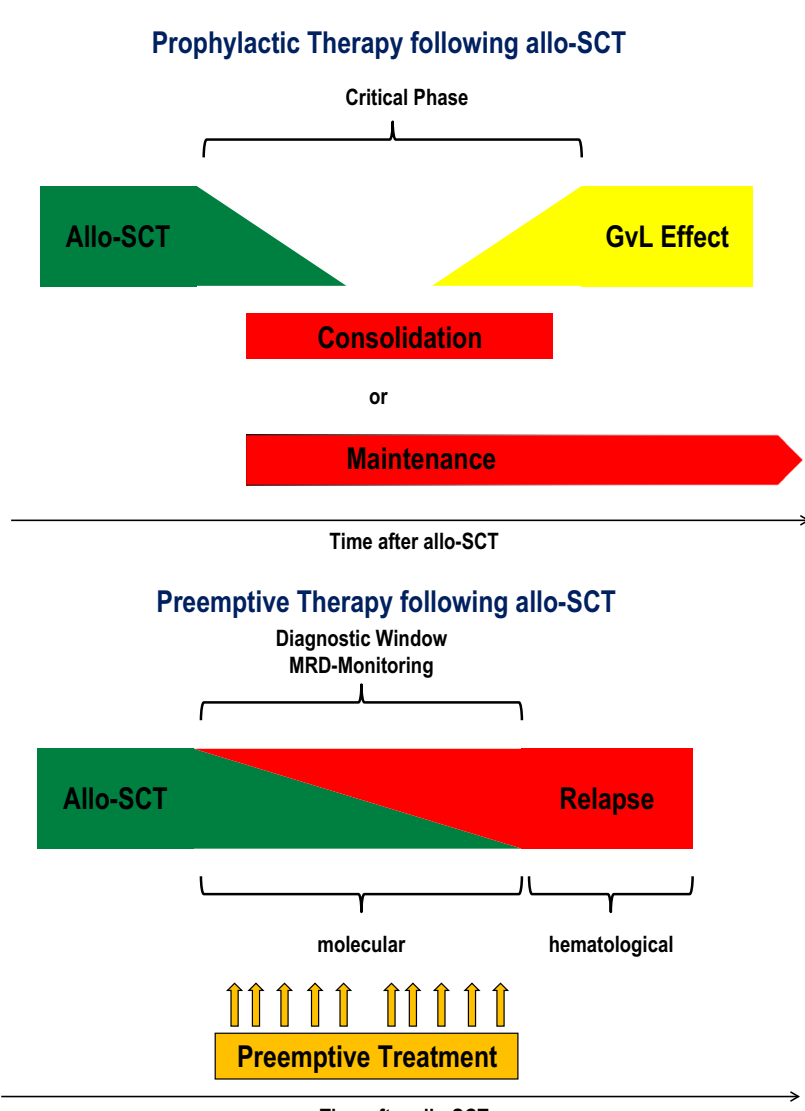

Time after allo-SCT

Fig. 1 Scheme of prophylactic and pre-emptive therapy

long-term cure since allo-SCT can be a potentially curative therapy already on its own. Therefore, for some patients, HMA given after transplant may represent over-treatment associated with potentially detrimental side effects such as cytopenias and infections as well as eventually even secondary malignancies in "already cured" patients.

Taking this into account, pre-emptive therapy instead of prophylactic treatment in remission may be a better strategy. Such pre-emptive approaches are initiated as soon as there is any evidence of relapse at a submicroscopical level to avoid conversion to frank haematological relapse.

Adhering to these definitions, no prospective maintenance trials have been published so far. Only one retrospective case series recently reported on a maintenance approach in 18 patients, who were envisaged to receive Aza continuously until progression or intolerability [40]. In contrast, 5 prospective single-arm studies have been performed so far investigating consolidation therapy with either Aza $(n=3)$ or DAC $(n=2)$ for patients with AML or MDS after allo-SCT [41-45]. These early-phase studies covered 130 patients and demonstrated feasibility. In addition, one aim of these studies was to identify the optimal dosage and schedule for future trials based. As most relapses occur within the first year after transplant, consolidation therapy was planned to start within the first 2-3 months after transplant in these studies (Table 2). Nevertheless, toxicity can be a relevant problem in this early phase after transplant and contributed to a treatment onset later than per-protocol in most trials. Furthermore, de Lima et al. and Craddock et al. reported a drop-of rate of 50 and $27 \%$ of enrolled patients prior to the first administration of Aza due to toxicity, patient wish or relapse [41, 42]. This indicates that the study population represents a selected group of patients. As expected from the known toxicity profile of these substances, hematotoxicity and infections were the most common adverse events related to the study drugs. Consequently, despite the use of dosages that were significantly lower than the approved dosages, only a minor fraction of patients could receive all of the envisaged treatment cycles. Taken together, these trials highlighted the potential risks of post-transplant cytotoxic therapy and the limited size of patients and the absence of a control arm do not allow a definitive ranking of efficacy and safety so far. Still, the study of de Lima et al. suggested a lower likelihood to develop chronic GvHD in patients receiving Aza maintenance therapy [42].

Finally, the Aza dose identified in the study of Lima et al. provided the basis for an ongoing randomized phase III trial investigating Azacitidine for relapse prevention after alloSCT in patients with myeloid malignancies (NCT00887068) [42]. This trial is currently recruiting patients and 246 patients will be randomized to receive Aza or placebo for 12 months after allo-SCT. Results from this trial will hopefully elucidate the impact of Aza-based consolidation on relapse risk and GvHD.

With regard to pre-emptive therapy, the use of Aza has been tested in a prospective trial reported by Platzbecker and colleagues. Here, pre-emptive Aza therapy in 20 patients with MDS and AML was triggered by falling donor chimerism in circulating CD34+ cells. Up to 4 cycles of Aza were started as soon as the CD34+ donor chimerism dropped below a threshold of $80 \%$, while patients were still in haematological remission. Despite an improvement of chimerism (> 80\%) in half of the patients, this early intervention was able to induce durable remissions only in $3(30 \%)$ of the responders and did not avoid progression towards haematological relapse in the majority of patients [46]. This was probably related to the limited number of Aza cycles, but in particular to the fact that DLI were not part of the protocol. Given the opportunity of sensitive methods to detect NPM1, the same group has applied this concept of pre-emptive Aza treatment also to this molecularly defined AML subgroup including 3 patients of them with MRD after allo-SCT [47].

The recent discovery of several distinctive gene mutations in patients with myeloid malignancies by genomic high-throughput techniques together with technical advances regarding PCR-based methods will enable a stringent MRD monitoring for the majority of patients. This will help to 


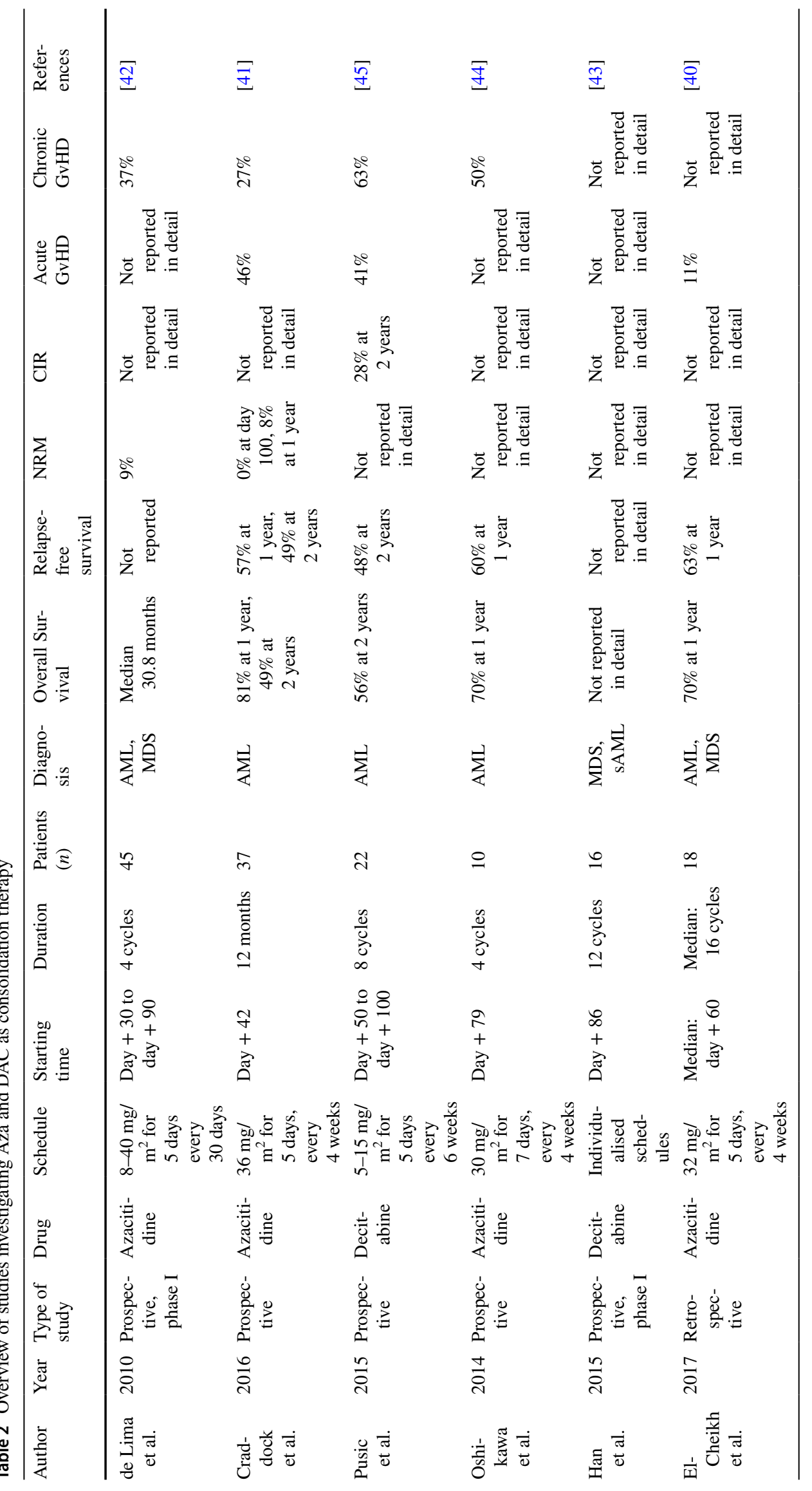


employ and optimize MRD-based pre-emptive therapies with HMA and other compounds in the close future.

\section{Mode of action}

To understand mechanisms by which HMA mediates its efficacy after allo-SCT animal models but also translational investigations of human samples have been exerted. In these analyses, HMA have shown to upregulate several antigens on leukemic cells in vitro and in vivo that were previously epigenetically silenced. This includes HLA epitopes, cancer testis antigens and minor histocompatibility molecules thought to render malignant cells more immunogenic toward T cell killing [48-51]. In further support of this, HMA seem to enhance T-cell mediated antitumor activity by increasing tumor-specific CD8 $\mathrm{T}$ cell responses against these upregulated molecules such as cancer testis antigens [52]. Another interesting mechanism seems to be the activation of endogenous retroviral elements (ERVs) through demethylation, which has been recently demonstrated in ovarian and colon cancer [53, 54]. By this 'viral mimicry', an interferon response in the tumor cells is induced resulting in an immune-mediated cancer cell killing.

Finally, cell surface expression of formerly unexpressed KIRs (killer Ig-like receptors) in natural killer (NK) cells is also modulated by HMA suggesting that interference with NK cell activity may also contribute to a HMA-mediated GvL effect [55].

The relatively infrequent and mostly mild GvHD observed in the majority of reports also supports the idea that HMA might offer immunoregulatory properties. In line with this, it was shown in mice that HMA convert conventional $\mathrm{T}$ cells to Tregs thereby preventing GVHD after allogeneic transplant or DLI without attenuating GVL. In these animals, HMA diminished GvHD severity and rate after allo-SCT and DLI resulted from direct suppression of T cell functionality and from conversion of allo-reactive donor $\mathrm{T}$ cells into Tregs (CD4+CD25+FOXP3+) through enhancement of FOXP3 expression [56, 57]. Correlating with this, Tregs seem to expand also in patients with AML and MDS during maintenance or salvage therapy after transplant [58, 59]. Taken together, these results suggest that HMA might target different immunological pathways and may hereby separate GvHD and GvL to a certain extent.

Besides these diverse immunomodulatory effects, another interesting mode of action could be related to the effects of HMA on the bone marrow microenvironment. We have recently demonstrated that mesenchymal stromal cells from patients with MDS and AML are aberrantly methylated [60-62]. Along with this, Verma et al. showed that Aza might mediate at least some of its efficacy via demethylation of the BM stroma [3].
Still, many of the underlying mechanisms need to be deciphered to gain a better understanding of the molecular and immunologic events associated with the use of HMA after allo-SCT.

\section{Practical issues}

Results from the retrospective reports and the limited number of prospective trials have established HMA and in particular Aza as a valuable treatment alternative for patients who relapse after allo-SCT [32, 33]. Still, several questions regarding the practical use of HMA as salvage therapy after transplant have not been answered sufficiently yet. To aid clinicians in their daily practice, we here comment on some of these issues based on the current knowledge and our own experience:

\section{Choice of HMA (Aza vs. DAC) for relapse after allo-SCT}

This question has not been addressed in randomized trials so far and the literature currently covers more patients treated with Aza than with DAC. Furthermore, DAC is only licensed for patients with AML but not MDS in Europe. For these reasons, we generally consider Aza as first choice in this setting and only use DAC in patients with contraindications against Aza or in case of Aza failure. However, both HMAs can induce remissions in patients relapsing after allo-SCT and the evidence regarding the use of DAC for relapse after allo-SCT is just growing. DAC might also be an alternative for AML patients with high blast counts or rapid disease kinetics at relapse. In addition, it was recently shown that patients exhibiting a TP53 mutation had an extraordinary high response rate to DAC [63]. Although this observation needs to be confirmed prospectively, it might be worth to consider DAC in this molecularly defined patient group.

\section{Dosing and schedule of HMA for treatment of relapse}

Table 1 depicts that different schedules with daily dosages ranging from 16 to $100 \mathrm{mg} / \mathrm{m}^{2}$ for 3-7 days have been used and were able to induce durable remissions. Indeed, no clear correlation between dosage and response has been found. Therefore, a definitive recommendation regarding dosage and schedule cannot be made. For patients with high leukemic burden or rapid relapse kinetics, one could assume that a higher dose might mediate a potentially stronger antileukemic effect. However, this should be balanced against potential side effects, in particular cytopenias and cytopeniarelated complications. 
We currently start with the approved Aza dosage of $75 \mathrm{mg} / \mathrm{m}^{2}$ for 7 days and adapt dosages during the following cycles in case of hematotoxicity. Similar to the situation in the non-transplant setting, we try to administer at least 4 cycles before a definitive evaluation of response can be made. In addition, duration of treatment is also not defined by any evidence from the literature. It remains unclear so far whether it is better to administer a definitive number of cycles or to continue until progression or intolerability as recommended in the non-transplant setting. Again, we follow the scheme of our prospective trial and administer 6-8 cycles of Aza if feasible and aim to infuse repetitive DLI until GvHD occurs. Since some of our patients experienced severe GvHD if DLI was the last intervention, we administer at least 1 cycle of Aza after the last DLI to take advantage of its assumed immunomodulatory effects.

\section{Are DLI and/or second transplant needed in addition to HMA?}

In case of relapse, after allo-SCT, it is a general principle to reduce disease burden by cytotoxic therapy and to combine this with a cellular approach to reinforce an allo-immune reaction. Amongst others, this principle has been exemplified by a large retrospective EBMT analysis. Here, Schmid and colleagues clearly demonstrated that re-induction of CR by pharmacological compounds alone is not sufficient for long-term survival, but donor-cell-based consolidation is required [64].

With regard to the use of DLI and second transplant HMA, the reports published so far were heterogeneous (Table 1) and give no answer whether a combination with donor cells is required for response and long-term survival. In the recent retrospective EBMT analysis, only those 39 patients who received DLI within 2 months of commencing AZA salvage and in the absence of a clinical response were included in multivariate analysis [16]. Probably as a result of this methodological limitation, the administration of DLI had no impact on either response or on 2-year overall survival in this register-based analysis. In contrast, in our retrospective analysis, $78 \%$ of the CR were obtained after the first DLI suggesting a pronounced cell-induced immune reaction. In further support of this idea, remissions induced by Aza and DLI in our analysis were durable in $66 \%$ of patients for a median time of 20 months and lasted for a median of 13 months even in those who finally relapsed again [22]. Finally, in the study of Platzbecker et al., DLI were not part of the protocol and probably relating to this pre-emptive Aza therapy could not avoid progression to frank hematologic relapse in the majority of patients [46].

Based on these considerations, we envisage combining Aza with DLI in all patients with relapsed AML and MDS. In those patients who achieve CR after this approach, we generally do not consolidate CR with a second transplant. We consider a second transplant only in those patients where no DLI are available or in patients who fail to Aza and DLI.

\section{Prophylactic or pre-emptive therapy with HMA?}

Results from randomized trials demonstrating a benefit of a prophylactic approach with HMA either as consolidation or maintenance are lacking so far. To us, prophylactic treatment might represent over-treatment associated with relevant side effects in a relevant proportion of patients. Thus, no recommendation for a prophylactic approach can be made and patients should be treated in clinical trials.

In the future, a relevant challenge will probably be risk stratification and to tailor post-transplant treatment based on the individual risk for relapse risk. Besides known risk factors such as remission state or karyotype knowledge about specific somatic mutations will also be incorporated into such stratification algorithms. Potential candidates for this seem to be TP53 mutations, as several analyses indicated a dismal prognosis for MDS patients after allo-SCT [65-68]. The goal is to identify a patient population with an extraordinary high relapse risk for further studies to test innovative prophylactic strategies after transplant. In patients with an intermediate risk for relapse, MRD-triggered pre-emptive therapy including DLI instead of treatment in remission may be a better strategy.

\section{Potential combination partners}

Several compounds such as HDAC- and tyrosine kinase inhibitors (TKI) or the immunomodulator Lenalidomide have been tested in combination with HMA in the nontransplant setting. Unfortunately, all of them have failed to improve response rate and survival when compared to monotherapy with HMA [69]. Nevertheless, based on early positive signal from one of these trials [70] some combinations are currently also under investigation for treatment of relapse after allo-SCT. Currently, the potentially additive effect of Lenalidomide is under investigation in 2 prospective trials (VIOLA trial and NCT02472691). The rationale for this combination is a potential stimulation of the donor immune system by Lenalidomide to potentiate the Azamediated GvL effect.

Several TKI with inhibitory effects on internal tandem duplications (ITD) in the gene encoding for the Fms-like tyrosine-3 (FLT3) kinase receptor are currently tested in clinical trials. Midostaurin will be the first one to be approved for conventional AML therapy [71]. For this reasons, TKI with FLT3-inhibiting activity have also been tested after allo-SCT. For example, therapy with Sorafenib, a multikinase inhibitor with activity against FLT3 kinase, has demonstrated antileukemic activity with or without DLI in this situation and 
can induce complete molecular remissions in some patients [72, 73]. Given the promising results of a recent phase-II trial combining Sorafenib and Aza in relapsed or refractory FLT3-ITD-mutated AML [74], we tested this combination based on an individual decision in 8 patients with relapsed FLT3+ AML after transplant. Following this combination, 4 patients achieved a complete remission (50\%) with two of them remaining in remission $>1$ year now without any antileukemic treatment [75].

The IDH2 inhibitor Enasidenib appears to be another very interesting compound for the post-transplant period. This small molecule mediates its effect rather by differentiation than by a direct cytotoxic effect against myeloid blasts and induces a promising response rate of $40 \%$ [76, 77]. Enasidenib has just recently been approved in the USA for the treatment of patients with IDH2-mutated relapsed/refractory AML. Based on its efficacy and its low toxicity, it seems reasonable to test Enasidenib as mono- or combination with HMA also in patients relapsing after allo-SCT.

Finally, there are early preliminary signals from in vitro and in vivo analyses suggesting that a combination of HMA and PD1-blocking agents may have a pathophysiological rationale in AML and MDS [78, 79]. Along with this, first reports suggest that immune checkpoint blockade may also be efficient in case of relapse after allo-SCT [80, 81].

\section{Conclusions}

HMA and in particular Aza have proven to be a valuable treatment for MDS and AML patients relapsing after allo-SCT and have consequently been integrated into clinical guidelines. To further optimize this approach, a better understanding of the underlying mechanisms and identification of target patient populations are required. Together with new pharmacological compounds, specialized cellular products and antibodies, this will hopefully help to further improve the prognosis of relapse after allo-SCT.

\section{Compliance with ethical standards}

Financial disclosure statement T S. had a consulting role for Celgene Corporation, Germany and received financial travel support and lecture fees from Celgene Corporation, Germany. T. S. also received financial travel support and lecture fees from Janssen-Cilag GmbH, Germany. C. R. received financial travel support from Celgene Corporation, Germany. R. H. and U. G. have nothing to declare. G. K. received financial travel support, research funding and lecture fees from Celgene Corporation, Germany.

\section{References}

1. Sureda A, Bader P, Cesaro S, Dreger P, Duarte RF, Dufour C, et al. Indications for allo- and auto-SCT for haematological diseases, solid tumours and immune disorders: current practice in Europe, 2015. Bone Marrow Transplant. 2015;50(8):1037-56.

2. Gooley TA, Chien JW, Pergam SA, Hingorani S, Sorror ML, Boeckh M, et al. Reduced mortality after allogeneic hematopoietic-cell transplantation. N Engl J Med. 2010;363(22):2091-101.

3. Bhagat TD, Chen S, Bartenstein M, Barlowe AT, Von Ahrens D, Choudhary GS, et al. Epigenetically aberrant stroma in MDS propagates disease via Wnt/beta-catenin activation. Can Res. 2017;77(18):4846-57.

4. de Lima M, Porter DL, Battiwalla M, Bishop MR, Giralt SA, Hardy NM, et al. Proceedings from the National Cancer Institute's second international workshop on the biology, prevention, and treatment of relapse after hematopoietic stem cell transplantation: part III. Prevention and treatment of relapse after allogeneic transplantation. Biol Blood Marrow Transplant. 2014;20(1):4-13.

5. Dombret H, Seymour JF, Butrym A, Wierzbowska A, Selleslag $\mathrm{D}$, Jang $\mathrm{JH}$, et al. International phase 3 study of azacitidine vs conventional care regimens in older patients with newly diagnosed AML with > 30\% blasts. Blood. 2015;126(3):291-9.

6. Fenaux P, Mufti GJ, Hellstrom-Lindberg E, Santini V, Finelli C, Giagounidis A, et al. Efficacy of azacitidine compared with that of conventional care regimens in the treatment of higher-risk myelodysplastic syndromes: a randomised, open-label, phase III study. Lancet Oncol. 2009;10(3):223-32.

7. Fenaux P, Mufti GJ, Hellstrom-Lindberg E, Santini V, Gattermann $\mathrm{N}$, Germing U, et al. Azacitidine prolongs overall survival compared with conventional care regimens in elderly patients with low bone marrow blast count acute myeloid leukemia. J Clin Oncol. 2010;28(4):562-9.

8. Kantarjian HM, Thomas XG, Dmoszynska A, Wierzbowska A, Mazur G, Mayer J, et al. Multicenter, randomized, open-label, phase III trial of decitabine versus patient choice, with physician advice, of either supportive care or low-dose cytarabine for the treatment of older patients with newly diagnosed acute myeloid leukemia. J Clin Oncol. 2012;30(21):2670-7.

9. Graef T, Kuendgen A, Fenk R, Zohren F, Haas R, Kobbe G. Successful treatment of relapsed AML after allogeneic stem cell transplantation with azacitidine. Leuk Res. 2007;31(2):257-9.

10. Czibere A, Bruns I, Kroger N, Platzbecker U, Lind J, Zohren F, et al. 5-Azacytidine for the treatment of patients with acute myeloid leukemia or myelodysplastic syndrome who relapse after allo-SCT: a retrospective analysis. Bone Marrow Transplant. 2010;45(5):872-6.

11. Jabbour E, Giralt S, Kantarjian H, Garcia-Manero G, Jagasia $\mathrm{M}$, Kebriaei $\mathrm{P}$, et al. Low-dose azacitidine after allogeneic stem cell transplantation for acute leukemia. Cancer. 2009;115(9):1899-905.

12. Lubbert M, Bertz H, Wasch R, Marks R, Ruter B, Claus R, et al. Efficacy of a 3-day, low-dose treatment with 5-azacytidine followed by donor lymphocyte infusions in older patients with acute myeloid leukemia or chronic myelomonocytic leukemia relapsed after allografting. Bone Marrow Transplant. 2010;45(4):627-32.

13. Schroeder T, Czibere A, Platzbecker U, Bug G, Uharek L, Luft T, et al. Azacitidine and donor lymphocyte infusions as first salvage therapy for relapse of AML or MDS after allogeneic stem cell transplantation. Leukemia. 2013;27(6):1229-35.

14. Antar A, Otrock ZK, Kharfan-Dabaja M, Salem Z, Aractingi S, Mohty M, et al. Azacitidine in the treatment of extramedullary relapse of AML after allogeneic hematopoietic cell transplantation. Bone Marrow Transplant. 2013;48(7):994-5.

15. Bolanos-Meade J, Smith BD, Gore SD, McDevitt MA, Luznik L, Fuchs EJ, et al. 5-azacytidine as salvage treatment in relapsed myeloid tumors after allogeneic bone marrow transplantation. Biol Blood Marrow Transplant. 2011;17(5):754-8.

16. Craddock C, Labopin M, Robin M, Finke J, Chevallier P, Yakoub-Agha I, et al. Clinical activity of azacitidine in patients who 
relapse after allogeneic stem cell transplantation for acute myeloid leukemia. Haematologica. 2016;101(7):879-83.

17. Drozd-Sokolowska J, Gil L, Waszczuk-Gajda A, Madry K, Piekarska A, Dutka M, et al. Azacitidine use after allogeneic stem cell transplantation-results from the polish adult leukemia group. Transpl Proc. 2016;48(5):1802-5.

18. Ghobadi A, Choi J, Fiala MA, Fletcher T, Liu J, Eissenberg LG, et al. Phase I study of azacitidine following donor lymphocyte infusion for relapsed acute myeloid leukemia post allogeneic stem cell transplantation. Leuk Res. 2016;49:1-6.

19. Inoue A, Kawakami C, Takitani K, Tamai H. Azacitidine in the treatment of pediatric therapy-related myelodysplastic syndrome after allogeneic hematopoietic stem cell transplantation. J Pediatr Hematol Oncol. 2014;36(5):e322-4.

20. Ishikawa T, Fujii N, Imada M, Aoe M, Meguri Y, Inomata T, et al. Graft-versus-leukemia effect with a WT1-specific T-cell response induced by azacitidine and donor lymphocyte infusions after allogeneic hematopoietic stem cell transplantation. Cytotherapy. 2017;19(4):514-20.

21. Kim SY, Cho SG, Cho BS, Kim MS, Eom KS, Kim YJ, et al. Azacytidine treatment after discontinuation of immunosuppressants in patients with myelodysplastic syndrome and relapse after allo-SCT at a single center. Bone Marrow Transplant. 2010;45(8):1375-6.

22. Schroeder T, Rachlis E, Bug G, Stelljes M, Klein S, Steckel NK, et al. Treatment of acute myeloid leukemia or myelodysplastic syndrome relapse after allogeneic stem cell transplantation with azacitidine and donor lymphocyte infusions - a retrospective multicenter analysis from the German Cooperative Transplant Study Group. Biol Blood Marrow Transplant. 2015;21(4):653-60.

23. Steinmann J, Bertz H, Wasch R, Marks R, Zeiser R, Bogatyreva L, et al. 5-Azacytidine and DLI can induce long-term remissions in AML patients relapsed after allograft. Bone Marrow Transplant. 2015;50(5):690-5.

24. Tessoulin B, Delaunay J, Chevallier P, Loirat M, Ayari S, Peterlin $\mathrm{P}$, et al. Azacitidine salvage therapy for relapse of myeloid malignancies following allogeneic hematopoietic SCT. Bone Marrow Transplant. 2014;49(4):567-71.

25. Woo J, Deeg HJ, Storer B, Yeung C, Fang M, Mielcarek M, et al. Factors determining responses to azacitidine in patients with myelodysplastic syndromes and acute myeloid leukemia with early post-transplantation relapse: a prospective trial. Biol Blood Marrow Transplant. 2017;23(1):176-9.

26. Haferlach T, Nagata Y, Grossmann V, Okuno Y, Bacher U, Nagae $\mathrm{G}$, et al. Landscape of genetic lesions in 944 patients with myelodysplastic syndromes. Leukemia. 2014;28(2):241-7.

27. Papaemmanuil E, Gerstung M, Bullinger L, Gaidzik VI, Paschka $\mathrm{P}$, Roberts ND, et al. Genomic classification and prognosis in acute myeloid leukemia. N Engl J Med. 2016;374(23):2209-21.

28. Itzykson R, Kosmider O, Cluzeau T, Mansat-De Mas V, Dreyfus $\mathrm{F}$, Beyne-Rauzy $\mathrm{O}$, et al. Impact of TET2 mutations on response rate to azacitidine in myelodysplastic syndromes and low blast count acute myeloid leukemias. Leukemia. 2011;25(7):1147-52.

29. Jung SH, Kim YJ, Yim SH, Kim HJ, Kwon YR, Hur EH, et al. Somatic mutations predict outcomes of hypomethylating therapy in patients with myelodysplastic syndrome. Oncotarget. 2016;7(34):55264-75.

30. Meldi K, Qin T, Buchi F, Droin N, Sotzen J, Micol JB, et al. Specific molecular signatures predict decitabine response in chronic myelomonocytic leukemia. J Clin Investig. 2015;125(5):1857-72.

31. Woo J, Howard NP, Storer BE, Fang M, Yeung CC, Scott BL, et al. Mutational analysis in serial marrow samples during azacitidine treatment in patients with post-transplant relapse of acute myeloid leukemia or myelodysplastic syndromes. Haematologica. 2017;102(6):e216-8. https://doi.org/10.3324/ haematol.2016.162909.
32. de Witte T, Bowen D, Robin M, Malcovati L. Allogeneic hematopoietic stem cell transplantation for MDS and CMML: recommendations from an international expert panel. Blood. 2017;129(13):1753-62.

33. Dohner H, Estey E, Grimwade D, Amadori S, Appelbaum FR, Buchner T, et al. Diagnosis and management of AML in adults: 2017 ELN recommendations from an international expert panel. Blood. 2017;129(4):424-47.

34. Ganguly S, Amin M, Divine C, Aljitawi OS, Abhyankar S, McGuirk JP. Decitabine in patients with relapsed acute myeloid leukemia (AML) after allogeneic stem cell transplantation (alloSCT). Ann Hematol. 2013;92(4):549-50.

35. Phillips CL, Davies SM, McMasters R, Absalon M, O'Brien $\mathrm{M}, \mathrm{Mo} \mathrm{J}$, et al. Low dose decitabine in very high risk relapsed or refractory acute myeloid leukaemia in children and young adults. Br J Haematol. 2013;161(3):406-10.

36. Singh SN, Cao Q, Gojo I, Rapoport AP, Akpek G. Durable complete remission after single agent decitabine in AML relapsing in extramedullary sites after allo-SCT. Bone Marrow Transplant. 2012;47(7):1008-9.

37. Liu XL, Zhao X, Wang C, Gao SJ, Tan YH. Decitabine treatment for acute myeloid leukemia relapse after allogeneic hematopoietic stem cell transplantation. J Biol Regul Homeost Agents. 2017;31(1):171-5.

38. Wang B, Jin X, Wang Q, Jing Y. Decitabine + CAG + DLI in relapsed acute myeloid leukemia after allogeneic stem cell transplantation. J BUON. 2016;21(1):280-1.

39. Schroeder T, Rautenberg C, Krüger W, Platzbecker U, Bug G, Steinmann J, Klein S, Hopfer O, Nachtkamp K, Kondakci M, Geyh S, Haas R, Germing U, Bornhäuser M, Kobbe G. Treatment of relapsed AML and MDS after allogeneic stem cell transplantation with decitabine and DLI -a retrospective multicenter analysis on behalf of the german cooperative transplant study group. Ann Hematol. 2017. (Accepted for publication)

40. El-Cheikh J, Massoud R, Fares E, Kreidieh N, Mahfouz R, Charafeddine $\mathrm{M}$, et al. Low-dose 5-azacytidine as preventive therapy for relapse of AML and MDS following allogeneic HCT. Bone Marrow Transplant. 2017;52(6):918-21.

41. Craddock C, Jilani N, Siddique S, Yap C, Khan J, Nagra S, et al. Tolerability and clinical activity of post-transplantation azacitidine in patients allografted for acute myeloid leukemia treated on the RICAZA trial. Biol Blood Marrow Transplant. 2016;22(2):385-90.

42. de Lima M, Giralt S, Thall PF, de Padua Silva L, Jones RB, Komanduri K, et al. Maintenance therapy with low-dose azacitidine after allogeneic hematopoietic stem cell transplantation for recurrent acute myelogenous leukemia or myelodysplastic syndrome: a dose and schedule finding study. Cancer. 2010;116(23):5420-31.

43. Han S, Kim YJ, Lee J, Jeon S, Hong T, Park GJ, et al. Modelbased adaptive phase I trial design of post-transplant decitabine maintenance in myelodysplastic syndrome. J Hematol Oncol. $2015 ; 8: 118$

44. Oshikawa G, Kakihana K, Saito M, Aoki J, Najima Y, Kobayashi $\mathrm{T}$, et al. Post-transplant maintenance therapy with azacitidine and gemtuzumab ozogamicin for high-risk acute myeloid leukaemia. Br J Haematol. 2015;169(5):756-9.

45. Pusic I, Choi J, Fiala MA, Gao F, Holt M, Cashen AF, et al. Maintenance therapy with decitabine after allogeneic stem cell transplantation for acute myelogenous leukemia and myelodysplastic syndrome. Biol Blood Marrow Transplant. 2015;21(10):1761-9.

46. Platzbecker U, Wermke M, Radke J, Oelschlaegel U, Seltmann F, Kiani A, et al. Azacitidine for treatment of imminent relapse in MDS or AML patients after allogeneic HSCT: results of the RELAZA trial. Leukemia. 2012;26(3):381-9. 
47. Sockel K, Wermke M, Radke J, Kiani A, Schaich M, Bornhauser M, et al. Minimal residual disease-directed preemptive treatment with azacitidine in patients with NPM1-mutant acute myeloid leukemia and molecular relapse. Haematologica. 2011;96(10):1568-70.

48. Almstedt M, Blagitko-Dorfs N, Duque-Afonso J, Karbach J, Pfeifer D, Jager E, et al. The DNA demethylating agent 5-aza2 -deoxycytidine induces expression of NY-ESO-1 and other cancer/testis antigens in myeloid leukemia cells. Leuk Res. 2010;34(7):899-905.

49. Atanackovic D, Luetkens T, Kloth B, Fuchs G, Cao Y, Hildebrandt $\mathrm{Y}$, et al. Cancer-testis antigen expression and its epigenetic modulation in acute myeloid leukemia. Am J Hematol. 2011;86(11):918-22.

50. Hambach L, Ling KW, Pool J, Aghai Z, Blokland E, Tanke $\mathrm{HJ}$, et al. Hypomethylating drugs convert HA-1-negative solid tumors into targets for stem cell-based immunotherapy. Blood. 2009; 113(12):2715-22.

51. Pinto A, Maio M, Attadia V, Zappacosta S, Cimino R. Modulation of HLA-DR antigens expression in human myeloid leukaemia cells by cytarabine and 5-aza-2'-deoxycytidine. Lancet. 1984;2(8407):867-8.

52. Goodyear O, Agathanggelou A, Novitzky-Basso I, Siddique S, McSkeane T, Ryan G, et al. Induction of a CD8+ T-cell response to the MAGE cancer testis antigen by combined treatment with azacitidine and sodium valproate in patients with acute myeloid leukemia and myelodysplasia. Blood. 2010;116(11):1908-18.

53. Chiappinelli KB, Strissel PL, Desrichard A, Li H, Henke C, Akman B, et al. Inhibiting DNA methylation causes an interferon response in cancer via dsRNA including endogenous retroviruses. Cell. 2017;169(2):361.

54. Roulois D, Loo Yau H, Singhania R, Wang Y, Danesh A, Shen SY, et al. DNA-demethylating agents target colorectal cancer cells by inducing viral mimicry by endogenous transcripts. Cell. 2015;162(5):961-73.

55. Santourlidis S, Trompeter HI, Weinhold S, Eisermann B, Meyer KL, Wernet $P$, et al. Crucial role of DNA methylation in determination of clonally distributed killer cell Iglike receptor expression patterns in NK cells. J Immunol. 2002;169(8):4253-61.

56. Choi J, Ritchey J, Prior JL, Holt M, Shannon WD, Deych E, et al. In vivo administration of hypomethylating agents mitigate graft-versus-host disease without sacrificing graft-versusleukemia. Blood. 2010;116(1):129-39.

57. Sanchez-Abarca LI, Gutierrez-Cosio S, Santamaria C, Caballero-Velazquez T, Blanco B, Herrero-Sanchez C, et al. Immunomodulatory effect of 5-azacytidine $(5$-azaC): potential role in the transplantation setting. Blood. 2010;115(1):107-21.

58. Goodyear OC, Dennis M, Jilani NY, Loke J, Siddique S, Ryan $\mathrm{G}$, et al. Azacitidine augments expansion of regulatory $\mathrm{T}$ cells after allogeneic stem cell transplantation in patients with acute myeloid leukemia (AML). Blood. 2012;119(14):3361-9.

59. Schroeder T, Frobel J, Cadeddu RP, Czibere A, Dienst A, Platzbecker U, et al. Salvage therapy with azacitidine increases regulatory $\mathrm{T}$ cells in peripheral blood of patients with AML or MDS and early relapse after allogeneic blood stem cell transplantation. Leukemia. 2013;27(9):1910-3.

60. Geyh S, Oz S, Cadeddu RP, Frobel J, Bruckner B, Kundgen A, et al. Insufficient stromal support in MDS results from molecular and functional deficits of mesenchymal stromal cells. Leukemia. 2013;27(9):1841-51

61. Geyh S, Rodriguez-Paredes M, Jager P, Khandanpour C, Cadeddu RP, Gutekunst J, et al. Functional inhibition of mesenchymal stromal cells in acute myeloid leukemia. Leukemia. 2016;30(3):683-91.
62. Schroeder T, Geyh S, Germing U, Haas R. Mesenchymal stromal cells in myeloid malignancies. Blood research. 2016;51(4):225-32.

63. Welch JS, Petti AA, Miller CA, Fronick CC, O'Laughlin M, Fulton RS, et al. TP53 and decitabine in acute myeloid leukemia and myelodysplastic syndromes. N Engl J Med. 2016;375(21):2023-36.

64. Schmid C, Labopin M, Nagler A, Niederwieser D, Castagna L, Tabrizi R, et al. Treatment, risk factors, and outcome of adults with relapsed AML after reduced intensity conditioning for allogeneic stem cell transplantation. Blood. 2012;119(6):1599-606.

65. Bejar R, Stevenson KE, Caughey B, Lindsley RC, Mar BG, Stojanov $\mathrm{P}$, et al. Somatic mutations predict poor outcome in patients with myelodysplastic syndrome after hematopoietic stem-cell transplantation. J Clin Oncol. 2014;32(25):2691-8.

66. Della Porta MG, Galli A, Bacigalupo A, Zibellini S, Bernardi M, Rizzo E, et al. Clinical effects of driver somatic mutations on the outcomes of patients with myelodysplastic syndromes treated with allogeneic hematopoietic stem-cell transplantation. J Clin Oncol. 2016;34:3627-37.

67. Lindsley RC, Saber W, Mar BG, Redd R, Wang T, Haagenson $\mathrm{MD}$, et al. Prognostic mutations in myelodysplastic syndrome after stem-cell transplantation. N Engl J Med. 2017;376(6):536-47.

68. Yoshizato T, Nannya Y, Atsuta Y, Shiozawa Y, Iijima-Yamashita Y, Yoshida K, et al. Genetic abnormalities in myelodysplasia and secondary acute myeloid leukemia: impact on outcome of stem cell transplantation. Blood. 2017;129(17):2347-58.

69. Sekeres MA, Othus M, List AF, Odenike O, Stone RM, Gore $\mathrm{SD}$, et al. Randomized phase II study of azacitidine alone or in combination with lenalidomide or with vorinostat in higher-risk myelodysplastic syndromes and chronic myelomonocytic leukemia: North American Intergroup Study SWOG S1117. J Clin Oncol. 2017;35(24):2745-53.

70. Sekeres MA, Tiu RV, Komrokji R, Lancet J, Advani AS, Afable $\mathrm{M}$, et al. Phase 2 study of the lenalidomide and azacitidine combination in patients with higher-risk myelodysplastic syndromes. Blood. 2012;120(25):4945-51.

71. Stone RM, Mandrekar SJ, Sanford BL, Laumann K, Geyer $\mathrm{S}$, Bloomfield CD, et al. Midostaurin plus chemotherapy for acute myeloid leukemia with a FLT3 mutation. N Engl J Med. 2017;377(5):454-64.

72. Metzelder SK, Schroeder T, Finck A, Scholl S, Fey M, Gotze $\mathrm{K}$, et al. High activity of sorafenib in FLT3-ITD-positive acute myeloid leukemia synergizes with allo-immune effects to induce sustained responses. Leukemia. 2012;26(11):2353-9.

73. Schroeder T, Zohren F, Saure C, Bruns I, Czibere A, Safaian NN, et al. Sorafenib treatment in 13 patients with acute myeloid leukemia and activating FLT3 mutations in combination with chemotherapy or as monotherapy. Acta Haematol. 2010;124(3):153-9.

74. Ravandi F, Alattar ML, Grunwald MR, Rudek MA, Rajkhowa T, Richie MA, et al. Phase 2 study of azacytidine plus sorafenib in patients with acute myeloid leukemia and FLT-3 internal tandem duplication mutation. Blood. 2013;121(23):4655-62.

75. Rautenberg C, Nachtkamp K, Dienst A, Schmidt PV, Heyn C, Kondakci M, et al. Sorafenib and azacitidine as salvage therapy for relapse of FLT3-ITD mutated AML after allo-SCT. Eur J Haematol. 2017;98(4):348-54.

76. Amatangelo MD, Quek L, Shih A, Stein EM, Roshal M, David MD, et al. Enasidenib induces acute myeloid leukemia cell differentiation to promote clinical response. Blood. 2017;130(6):732-41.

77. Stein EM, DiNardo CD, Pollyea DA, Fathi AT, Roboz GJ, Altman $\mathrm{JK}$, et al. Enasidenib in mutant IDH2 relapsed or refractory acute myeloid leukemia. Blood. 2017;130(6):722-31.

78. Orskov AD, Treppendahl MB, Skovbo A, Holm MS, Friis LS, Hokland M, et al. Hypomethylation and up-regulation of PD-1 
in T cells by azacytidine in MDS/AML patients: a rationale for combined targeting of PD-1 and DNA methylation. Oncotarget. 2015;6(11):9612-26.

79. Yang H, Bueso-Ramos C, DiNardo C, Estecio MR, Davanlou M, Geng QR, et al. Expression of PD-L1, PD-L2, PD-1 and CTLA4 in myelodysplastic syndromes is enhanced by treatment with hypomethylating agents. Leukemia. 2014;28(6):1280-8.
80. Albring JC, Inselmann S, Sauer T, Schliemann C, Altvater B, Kailayangiri S, et al. PD-1 checkpoint blockade in patients with relapsed AML after allogeneic stem cell transplantation. Bone Marrow Transplant. 2017;52(2):317-20.

81. Davids MS, Kim HT, Bachireddy P, Costello C, Liguori R, Savell A, et al. Ipilimumab for patients with relapse after allogeneic transplantation. N Engl J Med. 2016;375(2):143-53. 